\title{
Living-dwelling: the importance of half-private spaces in the neighbourhoods on the city border line
}

\author{
M. Zinoski, S. Solarska, I. Medarski \& O. Marina \\ Faculty of Architecture, Ss. Cyril and Methodius University, \\ Republic of Macedonia
}

\begin{abstract}
In order to perform a participatory action project within local community, it is necessary to establish research with social aspects significant for the residents of the particular neighbourhood. The key assumption of this participatory research project is founded on collaboration between residents, local authorities and experts and tends to emphasize the small scale of half-private space as a model of social sustainability. Considering the identity of living space, certain similarities arise as an important issue regarding the coherency of a built structure. Therefore, the importance of half-private space becomes an important aspect of a well-defined neighbourhood. This paper presents the research where students, dwellers and the local municipality - all stakeholders from different areas - work together on a participatory action. In order to fulfil the problematic of the usage of half private spaces, the neighbourhood on the border of the city is emphasized. The phenomena of emerging city border neighbourhoods appeared during the period of industrialization of the city of Skopje. Different schools from different areas of expertise are involved in the educational process because education nowadays has a global influence. Students have learned how to create and use analytical tools in the process of creating a program which has social significance for the inhabitants. A common topic was established - the importance of half-private spaces. In addition to the students, as a part in this project, the municipality and residents also benefit from this educational process. Governing in a smaller scale - part of the city - the municipality also becomes part of the transformation of a contemporary Balkan city. The residents can realize that their half-private spaces could have new social significance and become a social condenser.

Keywords: social condenser, half-private, city-border, neighbourhood.
\end{abstract}




\section{Introduction}

Since the urbanisation become a complex branch of space management, the participation of local community and their representatives influenced the creating of urban policy. This participatory collaboration between different stakeholders tends to become a sustainable concept in the process of creating the liveable and socially significant communities. By involving the different parties in the design process there is the assumption of creating an architectural concept with social significance for the final users. Depending on the standpoint, each party has a particular specific role regarding their participation in the whole process. The participatory actions in the architectural design process, considering the definition of architectural problems today, are commonly understood by assuming the user's needs and proposing an architectural concept. Usually it derives from previous experiences when defining the problem. Therefore, the purpose of this work is redefining the aprioristic approach which does not bring significant results for the local community regarding the sustainable concept as an estimative goal.

The research presented in this paper will give us the opportunity to realize how participatory actions in the local community, between residents, their representatives and experts in the area are inherent in defining the aspects of social sustainability in architectural research. To achieve these assets it is necessary to emphasize an academic and plausible methodology.

This paper presents research of social sustainability according to new proprietary conditions in transforming societies. It is important to emphasize that the research problem relates to aspects of the half private space in new properties. Since the agricultural land in rural areas near cities such as Skopje were transformed for housing, this immediately became the subject of urbanisation and under governance of local authorities. In the beginning of that process a vernacular type of housing with strong identity was starting to appear, and created a so-called "rurban" neighbourhood. Certain characteristics of rural and urban elements were recognised with strong social significance.

Thereby the purpose of research presented here is the plausibility of basic theoretical and methodological principles to achieve a social sustainability as a collaborative condition within communities.

The success of sustainable development programs in transitional societies is determined by their ability to achieve the highest attainable increase in living standards without measuring the least possible environmental and social degradation. This condition is present especially in the post socialist countries who are attempting to reach the European Union standards for living environment without acquiring certain knowledge. It seems that the local authorities and experts in that area of expertise emphasize environmental or economic sustainability on a macro level avoiding the aspects of social sustainability of the future development at the local level. There is a great possibility of "environmental degradation to occur in areas of high poverty and low social cohesion” [1, p. 3]. 


\section{Sustainable model}

The recent and most commonly used definition of sustainability, known as the Brundtland definition, established in the 1980s at the United Nations Commission on Environment and Development (UNCED) is: "Sustainable development is development that meets the needs of the present without compromising the ability of future generations to meet their own needs" [2].

For the successful implementation of sustainable development, the ability of members of a certain community to develop processes and structures which not only meet the needs of its current members but also support the ability of future generations to maintain a healthy and sustainable community is necessary.

There is great misinformation when defining sustainability regarding the context in which the definition is applied. Usually it is more important than its wording. Inclusive definitions may call for interdisciplinary input and a cohesive view of the interrelation of nature, society and the economy, but the primary goal of those who are performing the research - the stakeholders who would profit from its implementation - will quickly determine the real meaning of the work in the field of sustainability. Any community or organisation that concerns equal to environmental or economic sustainability should include social sustainability as a principle too. Social interactions within a community define the identity of the public domain and should be represented by authorities: "public domain is specified as a representation of collective needs. As an opposite of 'private' which etymologically means personification and unique, 'public' refers to selectivity and wholeness? The elements used to establish this aspect of collectivism and wholeness should be universal, understandable and familiar to their users. From the sociological point of view public authorities approach citizens and become their real representatives" [3]. In practice, this has not been the case. Therefore, the key aspect of social sustainability depends on societal conditions of community at its local level. In that sense Phillip Sutton's words are: "sustainability is not 'about' the integration of ecological, social and economic issues, nor is it 'about' widespread consultation, nor is it 'about' improving quality of life. It is about maintaining or sustaining something" [4]. To understand this concept there is a necessity to identify the focus on involving the local community.

UNESCO's MOST (Management of Social Transformations) project has conducted a series of case studies on cities, and the social policies that determine their social sustainability. The social sustainability of a city in this project is defined as: "Development and/or growth that is compatible with the harmonious evolution of civil society, fostering an environment conducive to the compatible cohabitation of culturally and socially diverse groups while at the same time encouraging social integration, with improvements in the quality of life for all segments of the population" [4]. Their focus on the local in all these matters is due to recognition that "the social sustainability of cities is affected not only by nationwide spatial policies, but also, if not chiefly, by policy decisions and implementation at the local level" [4]. It is very hard to develop sufficient frameworks for social sustainability on a macro-level without failure of social theory principles [4]. 
What is instead required is a focus on local policies and institutions, to build up "comparative knowledge" about the key factors that make urban policies successful. Particular societies in transition cannot be studied, sustained or altered through policy or institutional change without reference to the transformation of the space (local region) they occupy, such things as the allocation of civic space, street design, the location of services in relation to population, and so on. The principle of "best practice experience" became such a model for social sustainability research that it takes the focus away from the "scientific" measurement of a condition and emphasizes "comparative knowledge". By doing this it allows for a wide range of collaborative research projects to be considered under the heading of social sustainability and community integration. The criteria for inclusion as a "social sustainability project" are that the project must be innovative, effective, and sustainable [4].

Social sustainability may be viewed as a process, as well as a condition [1, p. 22]. Each local community has its particular condition where architectural research problem has its own characteristics. Thereby, each indicator of that condition becomes actions, which can be implemented by the community as a whole in order to increase or preserve its current level of sustainability over time. Participatory action on a methodological level is simply a matter of rephrasing the indicator that is developing a series of mechanisms for a community to collectively identify its strengths and needs.

There is methodology that gives a possibility to achieve a social sustainability as a collaborative condition within communities. Processes within communities that can achieve that condition are some activities as indicators of the condition. Steps towards their implementation are these aspects of the process:

- Equal access to key services such as health, education, transport, housing and recreation;

- The needs of future generations will not be disadvantaged by the activities of the current generation;

- System of community and cultural relations in which the positive aspects of disparate cultures are valued and protected, and in which cultural integration is supported and promoted when it is desired by individuals and groups in the same public space;

- Participation of citizens in the local municipality at a local level;

- Creating a system for transmitting awareness of social sustainability from one generation to the next - a sense of community responsibility for maintaining that system of continuity;

- Developing mechanisms for a community to fulfil its own needs where possible through community action.

According to McKenzie [1, p. 23] these aspects should improve the social sustainability processes on a local level. 


\subsection{The Skopje experience}

Social sustainability as a collaborative process within communities was a workshop topic realised in December 2013 by students and professors from the Faculty of Architecture in collaboration with the Municipality of the city of Skopje - Centre. The whole process of research of public needs, collecting data related to the regulations and proposing an architectural concept was a perfect opportunity for collaboration with the Municipality of the Centre within their on-going activities. The following is a description of the collaboration process between the two institutions:

Phase 1: Detection of problem and to "label" it: non-places;

The purpose of the first phase was to make the students recognize public spaces which are not used to their full potential.

Phase 2: Development of the problem, history of the place through the plans and level of their realization;

Students had to elaborate the problem, take pictures, and create a survey regarding a better public space. Stduents had to review a morphological transformation of the place through urban plans, how it was transformed during periods of time and the reason it became what it is today, a socalled "non-place".

Phase 3: Developing an architectural concept and labelling it: reprogramming/new public realities.

After determination of the problem, students had to propose the best architectural design that will improve the public space.

Phase 4: Accepting the most doable architectural concept according to the predetermined criteria and Municipality budget.

Phase 5: Implementing a project proposal according to the annual plans of the Municipality.

During the research process students were investigating the problems of local inhabitants regarding the occupation of their public space. This concept of dialectic relation between the students' capabilities for research in architecture and residents' needs, introduced the process with a social significance only when it emerges from the contexts where it actually belongs.

\section{Strategies of managing social sustainability: participatory actions research}

In an attempt to redefine the implementation of a sustainable concept we would like to explain plausible methodology which gives significant results to the local community. A crucial goal is to elaborate an approach based on the "principle of inquiry into the actual or proposed actions of people, by and with those affected", $[5$, p. 9$]$, to reach the social aspect of a particular group of people. 
It is an important consideration to realize how participatory actions in local community, between residents, their representatives and experts correlate with the aspects of social sustainability in architectural research processes.

The objective of this approach to collaborative thinking and social engagement is to eliminate socially irrelevant inquiry managed "from outside". Participation is defined as the interactive engagement of stakeholder groups viewed as "communities of interest" [5, p. 10].

Defining the applicative methodology it is important to establish a relevant problem regarding the social issue between all stakeholders involved in the process. Therefore managing and mediating the knowledge of different actors is only feasible when it is done according to the direction of the "history" behind the immediate situation [5, p. 9].

\subsection{Pedagogy of the sustainable process: according to Chevalier and Buckles}

The role of academic approach in participation projects is different from a conventional "pedagogical academic program". Researching skills usually means to ground thinking on assumed and previously gained knowledge. Research in the participatory action projects requires a different kind of skilfulness, commonly ignored in academic settings and knowledge assets. "Questions about the relevance and broader impact of course-based learning and disciplinary research are seldom asked and poorly answered. The role of students and researchers should acquire the skill to design questions and a process of inquiry that engages people involved in real events. This approach will engage knowledge created 'meaningful events', rather than producing 'eventless' documents. 'Skilful means' refers to any method or strategy that is helpful because it is attuned to the capacities, needs, and circumstances of the people involved." [5, pp. 6-9].

A new approach established in participatory action research should acquire field research and new concepts and practices to improve evidence-based research. This approach "tends to marginalize it from mainstream learning and academic research institutions and from public policy-making processes" [5, pp. 6-9]. The key aspect in this research should engage local people invited to contribute to decisions about projects by being part of a participatory action research process.

Pedagogical benefits for students in participatory action research projects are distinctive versus academic because being involved in the process where people create new knowledge and meaning. This process gives the opportunity for students to develop practical tools to delve into local culture and value systems of community life. This approach "overcomes these problems by creating flexibly structured processes that support inquiry and dialogue in context and across social and knowledge boundaries" [5, p. 10]. 


\section{Two systems of participatory action research: result-based management (rbm) and process management (pm)}

Methods adapted from psychology to engage social sustainability, provide unique answers to the crucial question of how to mobilize cultural values and systems of knowledge and learning. Techniques to ask questions such as Problem Domain and Social Domain, offer both simple and advanced ways to build on local knowledge and value systems, using methods that avoid fixed terms and ideas and make use of differences in language and culture [5, p. 11].

\subsection{Result-based management (rbm)}

A stakeholder such as public administration, as a party in participatory processes usually narrows their involvement only on ordering planning documentation and issuing building permission according to the law. Their contribution in organisational development uses a somewhat narrow range of methods to plan and manage projects.

These methods, especially in the public sector, involve managing for results formally known as result-based management (rbm). This method begins with setting expectations that reflect common goals and specific objectives. Then, activities are designed and managed to achieve the expected results [5, p. 12].

This approach to management, beside its rationality, lean on previous experience, lacks reason and brings rigidity into planning and management where "unpredictability and uncertainty prevail".

Considering planning where unpredictability and uncertainty exist, and with limited knowledge of key factors, leads to actions that are mechanical and linear. The planning process deprives people of the flexibility they need to achieve the desired results under changing circumstances. In complex situations, rbm: "produces closed-system plans that are too simple and that may hide reality behind defined goals and projects" [5, p. 13].

Nowadays, to act socially in management methods within the project and other planning processes have evolved to include critical reference groups (involving participants) in key stages of the project cycle. This method includes: goal definition, information gathering, project planning and implementation. As such, they inject "participatory principles" into the planning process.

\subsection{Process management (pm)}

Process management is a method of approach to planning that can be used in complex, unpredictable situations common in the fields of development and social change. As mentioned earlier, a transitional society's transformation of the properties of land and migration in the middle of the last century was a common situation.

As in medical practice, planning becomes a form of continuous thinking grounded in ongoing activities. The result is a series of working hypotheses to be 
tested "in the middle" of complex situations that have no clear start or end [5, p. 13].

The method allows stakeholders to pursue different goals or activities within a common project. Under such circumstances, the planning process must respond to many interests as a key aspect of public interest of a particular community. This concept helps planners by anticipating the precise events and activities to satisfy the larger goals and processes.

Process management, implies methods and techniques where plans are made at the right time and adapted to ongoing results, which means that each step of the process can create inputs for the design of later steps. These techniques are a key feature of process management that introduces the social dimension into project planning.

People can then apply new learning to social participatory action guided by practical wisdom and a sense of purpose already established in the pattern language of their existential space.

\section{Living-dwelling: a participatory action between students of architecture and the residents in the neighbourhood of "Ilinden" Municipality, Skopje}

Being one of the fast growing suburban areas of the city of Skopje, the Municipality of Ilinden has developed specific urban living and dwelling patterns that could serve as a sustainable model for future development of the city. Through observing and contact with the dwellers particular social and cultural habits regarding the usability was recognized in spatial organisation of the yards.

It was recognised that participation of residents as stakeholders is important during the research of socially sustainable environments. This research was conducted during the two workshops. The first session was realised in June 2014 where the objectives have been to create a participatory action between local authorities from Municipality of "Ilinden", students and mentors from UKIM Faculty of Architecture in Skopje and the residents from the neighbourhood in "Ilinden". Students in close communication with the residents and local administration have investigated the actual dwelling and living habits of the neighbourhood focusing their research on the residential urban block in "Ilinden". In the course of one week, the students fulfilled several tasks including:

Task 1: Students prepare a specific questionnaire to determine the social and spatial distinctive qualities of the place regarding its inhabitants and their particular style of living;

Task 2: Students observed the neighbourhood to realize the crucial social and habitual patterns of inhabitants related to their dwelling;

Task 3: Students conducted a survey to establish participants' perception of qualities of their neighbourhood. In order to be sure that they asked the correct questions, students created three groups of questions: statistical parameters, program and spatial patterns, and needs/preferences. There 
was a part of the questionnaires where residents had been asked to sketch the floor plan of their house and also the floor plan of the house they would like to live in;

Task 4: Inhabitants created their own statements according to their perception of the neighbourhood. Residents were asked about their previous experience of their current living environment. They were invited to describe in their own words what they liked most and least about their living places and write it in the questionnaire to share it with the rest of the participants, and also to try to sketch the floor plan of their house. To "imagine your ideal living space" - participants were asked to reflect their visions and expectations concerning their future habitat by trying to sketch a floor plan which represents their ideal living place;

Task 5: Representatives from the Municipality recognised the importance of establishing inhabitants' needs. At this point, with regards to the research that the students had made, the municipality got a clear image of what the problems and needs of this particular neighbourhood were;

Task 6: Students summarized the results and created (social diagrams) according to the survey;

Task 7: Students presented the results to the representatives from the Municipality of "Ilinden".

The following final considerations emerged from the experience of implementing the participatory action processes from this workshop:

- Students have been introduced to a specific research methodology to realise public collective needs.

- This concept of dialectic relation between the students' knowledge for research in architecture and dwellers' living habits, introduced the "culture of building" where architecture has a social significance only when it emerges from the contexts where it actually belongs.

- Students became aware of the role of architects as educators of residents regarding their perception of dwelling.

The purpose of the follow up second session of the workshop undertaken in October 2015 had been to create, recognize and conceptualize social behaviour beyond the half-private space. As a result, the participants have proposed possible scenarios where particular and specific social activities are taking place. In this workshop, process management methodology was applied with tools that support careful analysis of the actors involved in a project, the problems they are facing, and the options for action they may use to solve these problems and achieve their goals.

Since the primary goal was to improve the educational process, to ground inquiry in a social purpose and the intention to act socially, the participatory action in Skopje has been a collaboration between UKIM Faculty of Architecture in Skopje, lecturers from the University of POLIS in Tirana, lecturers from the 
University of Belgrade and the local authorities and dwellers of the municipality of Ilinden, Skopje.

The methodology proposed in this workshop was an open, complex system's approach to thinking, dialogue, and action with the aim to create a process with practical tools for collaborative inquiry and social engagement of residents.

The main goal of this participatory action has been the engagement between the residents and the students of architecture, and their collaboration in order to identify the needs of the inhabitants. Accordingly, there has been a pedagogical purpose behind the action, regarding the students and the residents who have been taking part in a social design process.

\section{Techniques}

The social analysis techniques as a result of the second session was created to reflect basic living patterns: what are the problems that people face, who are the actors or stakeholders affected by a situation or with the capacity to intervene, and what are the options for action? [5, p. 16].

The half-private spaces, a house's front yard that are meeting the public zone of the street, have been in the focus for reprogramming and restructuring of the neighbourhood landscape. Architectural concepts have been created by taking the following steps as a methodological strategy of the workshop.

Step 1: Educative and informative meeting in a form of a seminar was held where teachers and lecturers from different areas of expertise and different universities discussed the importance of the aspects of the half private spaces.

Step 2: Interactive meeting with residents where they have identified their spatial needs and have mapped the required facilities.

Step 3: Creating questionnaires in which the questions have been chosen in pursuance of getting the answers needed that helped the students understand the residents' needs and their habits.

Step 4: Architectural conceptualization of the residents' requirements (working on development of the new spatial concepts) that will redefine the semiprivate threshold between private/home and public/street space.

The survey has helped the students get the needed information in order to create sociograms, related to the activity diagrams, which represent the actual and the proposed situation in the neighbourhood regarding social interaction between residents and their interlocking of interests on different scales.

The activities between students and residents planned for this participatory action have been grouped in the following sequential phases:

Phase 1: Theoretical background - students have attended an opening ceremony at the Faculty of Architecture followed by lectures with a theoretical background of the particular location and subject of interest. 
Phase 2: Municipality visit - the theoretical discussion has moved to the Municipality Hall of Ilinden, through a process of familiarization with the Municipality and its attributes.

Phase 3: Meeting with the neighbours - the important part of participatory action is to bring different stakeholders together. The students, after meeting with the local dwellers of Ilinden, have been introduced to the local needs. On the other hand, the dwellers have learned about the importance of the half-private space as a zone of social interaction.

Phase 4: Preparing questionnaires - based on the previous knowledge gathered from the site, the students have prepared questionnaires regarding different topics, covering different areas of interest. The encounter between the students and the residents from the previous phase have been the first step towards the goal of creating a relevant questionnaire.

Phase 5: Collecting data from the questionnaires - each of the groups collected the needed data from the questionnaires, and based on their suggested topic have graphically represented the outcome of the answers.

Phase 6: Creating sociograms - the survey has helped the students get the needed information in order to create sociograms related to the activity diagrams, which represent the actual and the proposed situation in the neighbourhood regarding social interaction between the residents and their interlocking of interests on different scales.

Phase 7: Video files have been produced, based on the sociograms. The animated files tried to achieve the ultimate goal of creating and interpolating different scenarios on a single base, which are being generated by the use of half-private space in the neighbourhood.

These techniques provided a theoretical conclusion as a summary. If the private space is defined as a local world of inhabitants, and the public space as a global world of strangers, then the half private space can be defined as an in-between zone. Its importance as a sustainable model for future development of a neighbourhood becomes an integral part of the city - creating the street landscape of the passer-by and defining the image of the neighbourhood, yet only serving the dweller on a local scale.

\section{Conclusions}

The authors of this paper aimed at establishing a definition of sustainability in this case study; i.e. without compromising the ability of future generations to meet their own needs, the process of managing the project described in the above participatory activities has tried to resolve the complexity of stakeholder positions in contemporary community settings. Emphasizing the social content, general and specific objectives of the project have interacted and evolved during this process of implementation of sustainable methodology and has become the subject of negotiations and compromises, which have changed during the process.

The purpose of introducing process management as a technique was to incorporate collaborative inquiry between academic assets into ongoing activities 
of local authorities, preserve living and dwelling patterns to make them sustainable within the neighbourhood at the same time. The idea to emphasize the living habits to be sustained is fulfilled through the steps of preservation and learning from a "living" neighbourhood inherited with social significance. Assessments are made for accounting purposes but also to guide social action of dwellers in circumstances that evolve over time to meet the unexpected needs of future generations.

As mentioned at the beginning, the elements used to establish this aspect of collectivism and coherence should be understandable and familiar to their users. Social sustainability becomes a technique where the planning process must respond to many interests as a key aspect of public interest of particular community reinforcing the connection between citizen and local authorities as their real representatives.

\section{References}

[1] McKenzie, S., Social Sustainability: Towards Some Definition, Hawke Research Institute University of South Australia: Magill, 2004.

[2] World Commission on Environment and Development, Our Common Future, Oxford University Press: Oxford, p. 2, 1987.

[3] Davis, H., The Culture of Building, Ars Lamina: Skopje, p. 46, 2006.

[4] Green Innovations Inc. \& Sutton, P., Sustainability: What Does it Mean? 2000. www.green-innovations.asn.au/sustblty.htm.

[5] Chevalier, J.M. \& Buckles, D.J., A Guide to Collaborative Inquiry and Social Engagement, Sage Publications: New Delhi, 2008. 